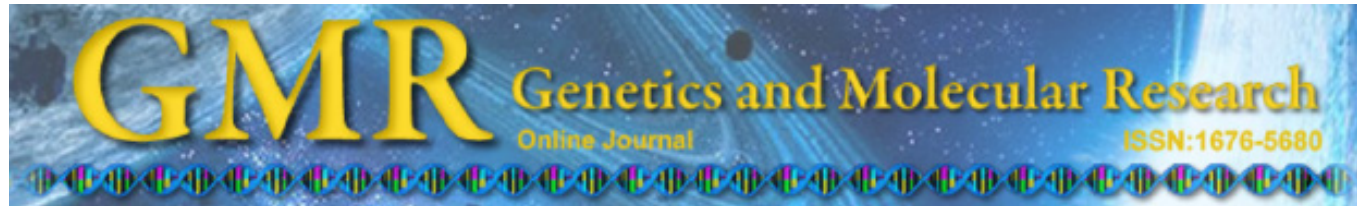

\title{
Development of a novel and efficient strategy for practical identification of Pyrus spp (Rosaceae) cultivars using RAPD fingerprints
}

\author{
J. Lin ${ }^{1}$, X.C. Wang ${ }^{2,3}$, Y.H. Chang' ${ }^{1}$ and J.G. Fang ${ }^{2,3}$ \\ ${ }^{1}$ Institute of Horticultural, Jiangsu Academy of Agricultural Sciences, \\ Nanjing, Jiangsu, P.R. China \\ ${ }^{2}$ College of Horticulture, Nanjing Agricultural University, \\ Nanjing, Jiangsu, P.R. China \\ ${ }^{3}$ Fruit Crop Genetic Improvement and Seedling Propagation Engineering \\ Center of Jiangsu Province, Nanjing, P.R. China \\ Corresponding author: Y.H. Chang \\ E-mail: cyh@jaas.ac.cn
}

Genet. Mol. Res. 10 (2): 932-942 (2011)

Received October 11, 2010

Accepted November 17, 2010

Published May 24, 2011

DOI 10.4238/vol10-2gmr1097

\begin{abstract}
Accurate and reliable cultivar identification of crop species is essential to guarantee plant material identity for purposes of registration, cultivar protection and production. To facilitate identification of plant cultivars, we developed a novel strategy for efficient recording of DNA molecular fingerprints in genotyped plant individuals. These fingerprints can be used as efficient referential information for quick plant identification. We made a random amplified polymorphic DNA (RAPD) marker analysis of 68 pear cultivars. All pear genotypes could be distinguished by a combination of eight 11-mer primers. The efficiency of the method was further verified by correct identification of four cultivars randomly chosen from the initial 68. The advantages of this identification include use of fewer primers and ease of cultivar separation by the corresponding primers marked on the cultivar identification diagram. The cultivar identification diagram can efficiently serve for pear cultivar identification by readily providing the information
\end{abstract}


needed to separate cultivars. To the best of our knowledge, this is the most efficient strategy for identification of plant varieties using DNA markers; it could be employed for the development of the pear industry and for the utilization of DNA markers to identify other plant species.

Key words: Pear; RAPD; Cultivar identification; Molecular markers

\section{INTRODUCTION}

Cultivar identification and characterization are the first steps of any fruit introduction and improvement program. Breeding efforts would be facilitated by information on the genetic diversity of available germplasm resources, such as lines available from commercial seed sources. Also, an effective method for cultivar identification and fingerprinting is essential for intellectual property protection and for early identification of seedlings in the nursery industry.

Pear (Pyrus spp) is an important fruit crop worldwide. It belongs to the genus Pyrus, which comprises at least 22 species, among which $P$. communis (the European pear) and $P$. pyrifolia and P. serotina (Asian pear or nashi) are the most utilized for fruit production. These species are diploid $(2 \mathrm{X}=34)$ and self-incompatible, resulting in great genetic variability within the species. More than 5000 cultivars exist today, although only a small percentage are commercially cultivated (Bell et al., 1996). The existence of a very large number of rootstocks, cultivars and clones maintained by vegetative propagation reinforces the need for a reliable method of pear cultivar identification for researchers, nurserymen and growers. This is a very important issue for the fruit industry, particularly since the sale of fruit trees and planting of orchards involve major investments in time and money.

Traditional identification, which employs morphological and physiological traits, such as leaf size, maturity time and seed shape, is largely influenced by environmental conditions and usually requires that plants be grown to maturity. Assessment of these traits is also difficult and their evaluation can be subjective considering that most of these cultivars are related. In addition, evaluation of morphological characters is very time consuming and results are not readily transferred from one environment to another (Baird et al., 1996). In the recent past, biochemical markers, such as isozymes and seed proteins, have been widely used, although their use has often been limited by the low frequency of polymorphisms in many plant species. The number of isozyme systems required increases with the number of cultivars to be evaluated.

The advent of DNA markers has led to their application in the identification of plant cultivars, which is one of the main uses of DNA marker. Unlike biochemical markers with limited polymorphism and spatial-temporal variations, DNA-based makers are not affected by physiology or the environment and can be used to identify cultivars at any stage of development. Recently, various DNA-based markers have been developed and used for studies in genetic diversity, relationships, and origins of cultivars (D'Onofrio et al., 2009; Melgarejo et al., 2009; Cheng and Huang, 2009; Elidemir and Uzun, 2009; Papp et al., 2010). Among these markers, random amplified polymorphic DNA (RAPD) (Williams et al., 1990) markers are useful for cultivar analysis, with advantages of simplicity, efficiency, relative ease of execution, and non-requirement of any previous sequence information. RAPD markers have been used in cultivar identification and genetic relationship analysis of a number of fruit species, such as apple (Stark-Urnau, 2002a), litchi (Ding et al., 2000), grape (Qu et al., 1996; Corazza- 
Nunes et al., 2002), cherry (Demirsoy et al., 2008), Indian cashew (Archak et al., 2003), longan (Yonemoto et al., 2006), olive (Belaj et al., 2003), pear (Stark-Urnau, 2002b; Lee et al., 2004), and pistachio (Kafkas et al., 2006). Despite all these advantages, there is no a single-efficient strategy for applying DNA markers in cultivar identification. The main reason for this situation is a dearth of practical analysis strategies for DNA fingerprints. The popular analysis techniques for DNA banding patterns known as cluster analyses are not efficient in cultivar or species separation. This has made the practice of utilizing DNA markers in crop and seed identification unpopular.

We developed a new strategy that makes the identification of pear cultivar a practical, efficient and reliable.

\section{MATERIAL AND METHODS}

\section{Plant materials}

Young leaves of 68 selected pear cultivars were collected from the Jiangsu Academy of Agricultural Sciences, Jiangsu, China (Table 1). The RAPD primers were synthesized by Shanghai Invitrogen Biotechnology company.

\section{Table 1. Name and origin of the pear cultivars separated using RAPD fingerprints.}

\begin{tabular}{|c|c|c|c|c|c|}
\hline No. & Cultivar & Origin & No. & Cultivar & Origin \\
\hline 1 & Eli 2 hao & Hubei & 35 & Wujiuxiang & Liaoning \\
\hline 2 & Xizilv & Zhejiang & 36 & Jinfeng & Liaoning \\
\hline 3 & Cili & Shandong & 37 & Zaosu & Liaoning \\
\hline 4 & Meigetsu & Japan & 38 & Wakahikari & Japan \\
\hline 5 & Cuiguan & Guangxi & 39 & Jinli & Shanxi \\
\hline 6 & Manao & Henan & 40 & Huanghuali & Zhejiang \\
\hline 7 & Chikusui & Japan & 41 & Yulvxiang & Shanxi \\
\hline 8 & Yaguangli & Hebei & 42 & Jinshui 2 hao & Hubei \\
\hline 9 & Hosui & Japan & 43 & Chonghuadali & Hubei \\
\hline 10 & Hanareum & Southkorea & 44 & Pingguoli & Liaoning \\
\hline 11 & Kousui & Japan & 45 & Long 19 & Sichuan \\
\hline 12 & Jinchuanxueli & Sichuan & 46 & Zuijinxiang & Liaoning \\
\hline 13 & Hanxiang & Jilin & 47 & Zaomeisu & Liaoning \\
\hline 14 & Jinhua 4 hao & Sichuan & 48 & Longquansuli & Sichuan \\
\hline 15 & Weiningdahuang & Guizhou & 49 & Dangshansuli & Anhui \\
\hline 16 & Starkrimson & USA & 50 & Jinhua & Sichuan \\
\hline 17 & Korla xiangli & Xinjiang & 51 & Xinyali & Hebei \\
\hline 18 & Jinshui 3 hao & Hubei & 52 & Zaojinxiang & Liaoning \\
\hline 19 & Bayuexue & Hebei & 53 & Jinxingli & Henan \\
\hline 20 & Qiyuesu & Henan & 54 & Guiguan & Zhejiang \\
\hline 21 & Huajin & Liaoning & 55 & Aixiang & Liaoning \\
\hline 22 & Zaohuang & Neimenggu & 56 & Nangetsu & Japan \\
\hline 23 & Beixin & Liaoning & 57 & Kisui & Japan \\
\hline 24 & Shinsei & Japan & 58 & Shinseiki & Japan \\
\hline 25 & Kikusui & Japan & 59 & Huawang & Shandong \\
\hline 26 & Nanguoli & Liaoning & 60 & D’Anjou & USA \\
\hline 27 & Zhongcui & Hubei & 61 & Jinzhui & Hebei \\
\hline 28 & Hongxiangmi & Henan & 62 & Hougetsu & Japan \\
\hline 29 & Okusankichi & Japan & 63 & Puli & Xinjiang \\
\hline 30 & Huasu & Liaoning & 64 & Bayuehong & Shanxi \\
\hline 31 & Yali & Hebei & 65 & Jinshui 1 hao & Hubei \\
\hline 32 & Cuilu & Zhejiang & 66 & Zhe 21 & Zhejiang \\
\hline 33 & Xuehuali & Hebei & 67 & Hangqing & Zhejiang \\
\hline 34 & Huangguan & Hebei & 68 & Aigan juicy & Japan \\
\hline
\end{tabular}




\section{Genomic DNA extraction and amplification of RAPD markers}

Total genomic DNA of each genotype was extracted from young leaves using the modified cetyltrimethylammonium bromide (CTAB) method (Murray and Thompson, 1980; Bousquet et al., 1990; Fang et al., 2006). The extracted DNA was diluted with $1 \mathrm{X}$ TE buffer to a final concentration of $10 \mathrm{ng} / \mu \mathrm{L}$ and stored at $-20^{\circ} \mathrm{C}$, pending use.

The reaction mixture (final volume $15 \mu \mathrm{L}$ ) contained $1.5 \mu \mathrm{L} 10 \mathrm{X}$ buffer, $1.2 \mu \mathrm{L}$ $\mathrm{MgCl}_{2}(25 \mathrm{mM}), 1.8 \mu \mathrm{L}$ dNTP $(2.5 \mathrm{mM}), 1.2 \mu \mathrm{L}$ primer $(1.0 \mu \mathrm{M}), 0.08 \mu \mathrm{L}$ rTaq polymerase DyNAzyme $(5 \mathrm{U} / \mu \mathrm{L})$ and $30 \mathrm{ng}$ genomic DNA. Amplification reactions were performed based on the standard protocol of Williams et al. (1990), with minor modifications. The polymerase chain reaction (PCR) was carried out in an Autorisierter Thermocycler (Eppendorf, Hamburg, Germany), programmed as follows: pre-denaturation for 5 min at $94^{\circ} \mathrm{C}$, then 42 cycles each consisting of a denaturation step for $30 \mathrm{~s}$, an annealing step for $1 \mathrm{~min}$ at annealing temperature (Table 2), and an extension step for $2 \mathrm{~min}$ at $72^{\circ} \mathrm{C}$. Amplification was terminated by a final extension of $10 \mathrm{~min}$ at $72^{\circ} \mathrm{C}$.

Table 2. Primers chosen for further fingerprinting of 68 pear genotypes.

\begin{tabular}{llc}
\hline Primer & Nucleotide sequence $\left(5^{\prime}-3^{\prime}\right)$ & Annealing temperature $\left({ }^{\circ} \mathrm{C}\right)$ \\
\hline Y6 & GTTTCGCTCCC & 44.4 \\
Y17 & AGGGGTCTTGG & 36.6 \\
Y27 & GTGTGCCCCAA & 44.4 \\
Y40 & AGCGTCCTCCT & 43.7 \\
Y46 & ACGACCGACAT & 44.4 \\
Y47 & ACGACCGACAG & 44.8 \\
Y48 & ACGACCGACAC & 44.8 \\
Y60 & ACCCCCGACTC & 43.7 \\
\hline
\end{tabular}

\section{RAPD analysis}

Fifty-four 11-mer RAPD primers were screened. In order to increase the credibility of the fragments, we scored only those that were very reproducible. As a result, only eight primers (Table 2) that showed well-resolved and reproducible bands were further analyzed; the rest were discarded. The PCR products were detected on $1.3 \%(\mathrm{w} / \mathrm{v})$ agarose gels in $1 \mathrm{X}$ TAE (0.04 M Tris-acetate, $0.001 \mathrm{M}$ EDTA, pH 8.0) buffer at $100 \mathrm{~V}$. The gels were stained with $0.5 \mu \mathrm{g} / \mathrm{mL}$ ethidium bromide and photographed under ultraviolet light. Polymorphic bands among the cultivars were observed from the photographs. In order to have reproducible and clear banding patterns, all amplifications were each repeated at least thrice.

\section{Data analysis}

Only the reproducible and clear-cut polymorphic RAPD profiles were manually scored from photographic gel prints of each cultivar, and specific bands were chosen to identify the cultivars. Cultivars sharing the same band patterns were clustered into the same subgroup, and then more primers were employed to distinguish the cultivars in each sub-group. As more primers were used, more and more amplified specific bands were generated, and 
eventually all the cultivars could be differentiated. Based on this, the cultivar identification diagram (CID), comprised of the bands with specific sizes used to separate the cultivars and all the related primers that generated the specific bands, was constructed for full separation of all the pear cultivars.

\section{Test of utilization and workability of the diagram in cultivar identification}

Two groups of pear cultivars were randomly chosen to verify the utilization and workability of the diagram showing the separation of the 68 cultivars. Corresponding primers that could amplify the polymorphic fragments to be used for separation of the cultivars could be readily found in the diagram. If the randomly chosen cultivars could be distinguished accurately and quickly as per the anticipated results on the whole CID, we can assure the workability and efficiency of this new approach in the identification of cultivars both for this study and also for similar study in the future. The data of the cultivar separation from this diagram can also be transferred to a database for ease of reference.

\section{RESULTS}

\section{Cultivar identification}

To establish a stable and optimistic RAPD system with high reproducibility, longer primers (11 nt) were employed, and the annealing temperatures for each primer were screened based on the quality and reproducibility of banding patterns. The primers were randomly screened from a stock of 5411 -mer primers, and once an optimal primer that could produce reproducible polymorphic bands was screened, it was utilized further in the identification of pear cultivars. After the eighth primer (Table 2) was screened and utilized, all the 68 pear cultivars could be successfully identified. Among the eight primers used, primer Y47 (Figure 1) was the first to be screened and used in identification of all the 68 pear cultivars. The electrophoresis results show that primer Y47 generated uniform, clear, and reproducible band patterns with a 1400-bp size in 42 cultivars. The lane numbers correspond to the codes in Table 1 . This group of cultivars was easily differentiated from the other 26 cultivars by the presence or absence of a distinct 1400-bp band, causing all the 68 cultivars to be separated into two groups (Figure 2). Following this, the second primer Y6 (Figure 2) was chosen to further differentiate members in the two resulting groups of pear cultivars. Primer Y6 could separate the two groups of cultivars identified by primer Y47 into smaller groups. The two initial groups, comprised of 42 and 26 pear cultivars, could both be separated into two subgroups each with a band size of about $1800 \mathrm{bp}$ (Figure 2). The remaining six primers (Table 2) were, step by step, screened and chosen to differentiate the pear cultivars until full separation was achieved (Figure 2). Eventually, all the 68 pear cultivars were successfully differentiated from each other by the joint use of eight different primers; the flow diagram of amplification makes the identification of these 68 cultivars an efficient, reliable, and simple process, useful for the pear industry, since there was a close connection between the specific bands, primers used and the cultivars identified. 


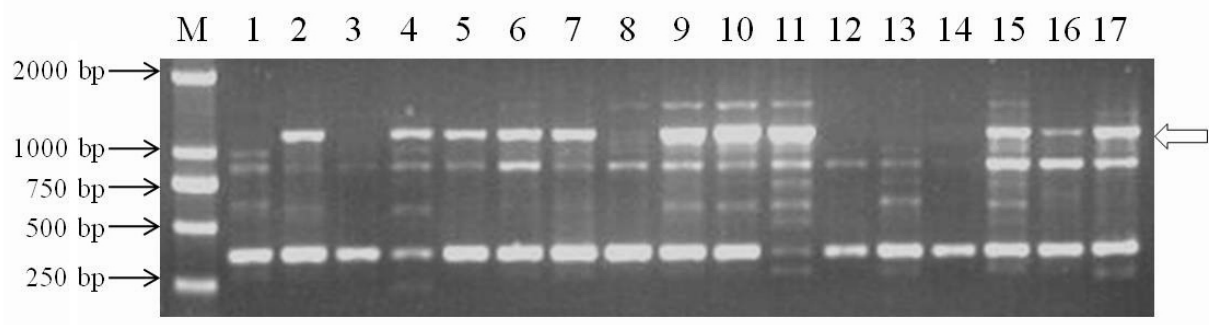

M $18 \quad 19202122 \quad 23242526 \quad 2728 \quad 29 \quad 30 \quad 31 \quad 32 \quad 33 \quad 34$

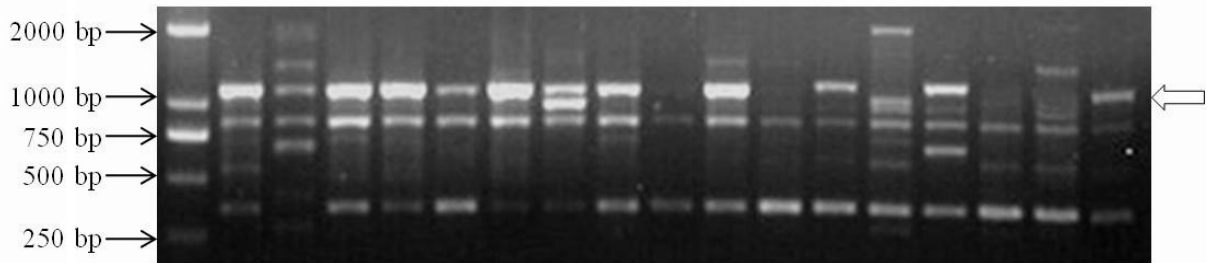

M $35 \quad 36 \quad 37 \quad 38 \quad 39 \quad 40 \quad 41 \quad 42 \quad 43 \quad 44 \quad 45 \quad 46 \quad 47 \quad 48 \quad 49 \quad 50 \quad 51$

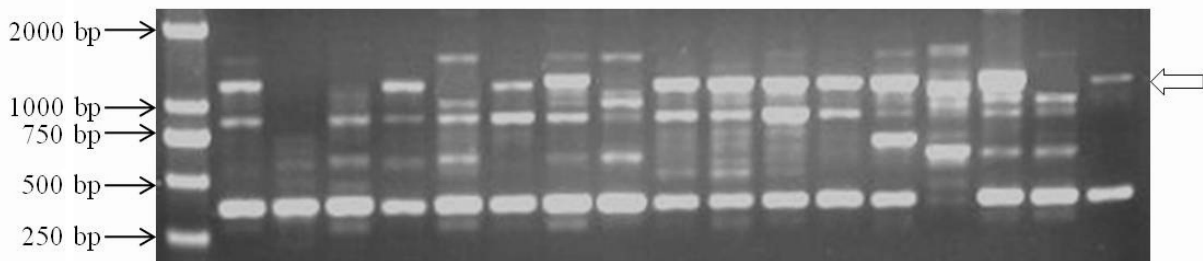

M $525335455 \quad 5657585960 \quad 61 \quad 62 \quad 63 \quad 64 \quad 65 \quad 66 \quad 67 \quad 68$

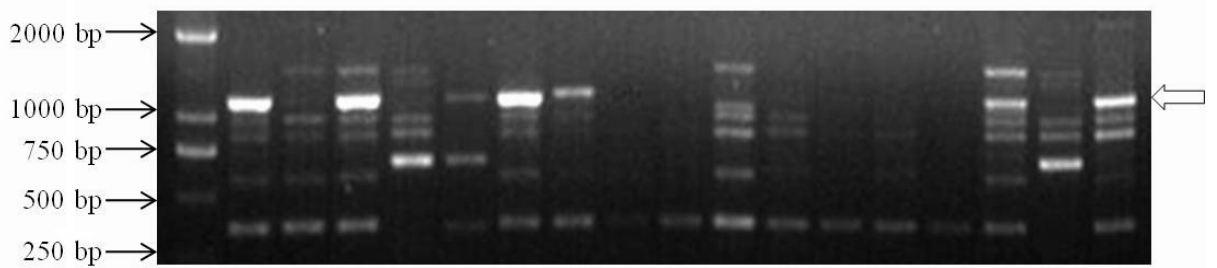

Figure 1. RAPD patterns of 68 genotypes within the genus Pyrus obtained with primer Y47. Horizontal arrows indicate the specific bands. The lane numbers correspond to the codes in Table 1. M=DNA size marker.

\section{Test of use and workability of the diagram in cultivar identification}

This strategy could also make DNA markers more applicable for plant variety identification. However, this study was not only aimed at generating a diagram like for cluster analysis of some cultivars; the diagram generated should be reliable and applicable for the practical identification of other pear cultivars even in future. To ascertain if this important objective had been met, verification of the utilization, workability and efficiency of the diagram in cultivar identification was done; two groups of cultivars including 'Huasu', 'Nanyue', 'Bayuehong', and 'Aiganshui' were randomly chosen to be used for the verification exercise. From the location of these cultivars in CID, it was easy to find the primer to use in separating them; the primers Y47, Y40 and Y27 were used to separate the four cultivars. PCR results show that the four pear cultivars could initially be separated into two groups by primer Y47, with a band of about $1400 \mathrm{bp}$. One group 
had 'Nanyue' and 'Aiganshui', and could further be separated by primer Y27, with the 1900-bp band. The other group was made up of 'Huasu' and 'Bayuehong', and could be divided by use of primer Y40, with a band of about $1200 \mathrm{bp}$ (Figure 3). Identification of the selected four cultivars using the three specific primers as anticipated indicates the usability and power of this CID. In this way, all the 68 cultivars could be successfully identified with suitable primer combinations.

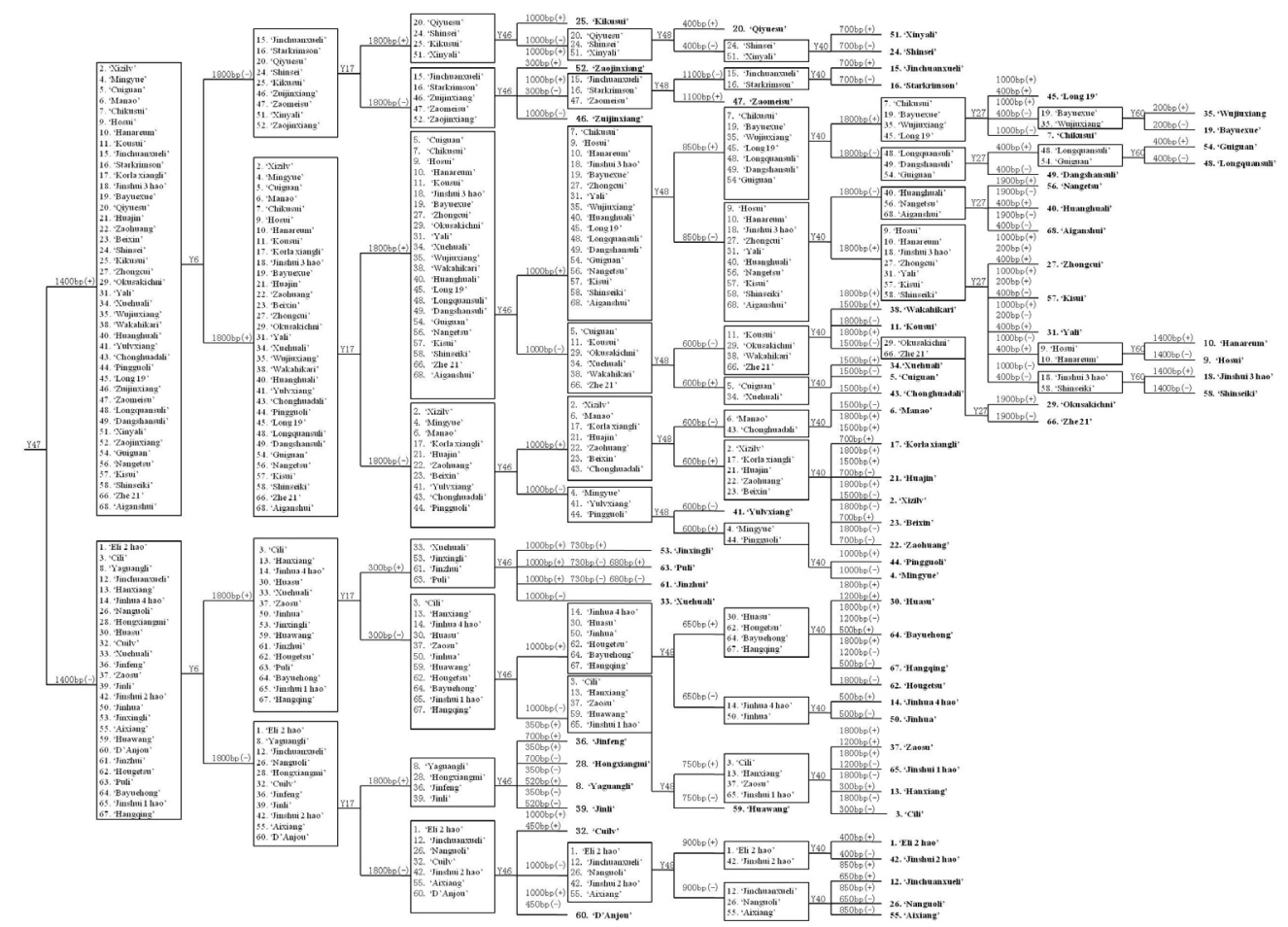

Figure 2. Cultivar identification diagram of 68 pear cultivars obtained with eight RAPD primers. Note: $(+)$ band present; (-) band absent. The names of the cultivars in bold mean that these cultivars were completely separated.

A

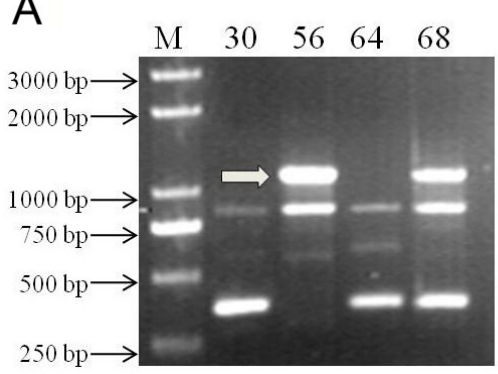

B

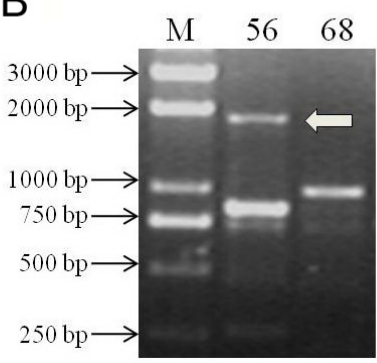

C

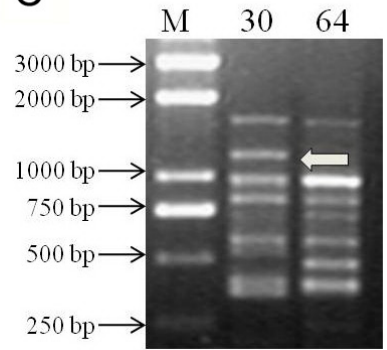

Figure 3. RAPD profiles obtained with RAPD primers. Horizontal arrows indicate the specific bands. The lane numbers correspond to the codes in Table 1. $\mathrm{M}=$ DNA size marker. A. Obtained with primer Y47. B. Obtained with primer Y27. C. Obtained with primer Y40. 


\section{DISCUSSION}

One of the main purposes of plant science is to provide service to agriculture, with the application of new biological techniques to agricultural practices being very important. Even though several generations of DNA markers have been developed and used for cultivar identification (Saker et al., 2006; Chiu et al., 2010), genetic analysis (Boronnikova et al., 2007; Silvestrini et al., 2008; Bhau et al., 2009; Baysal et al., 2010), and in the process of thousands of papers published, they are not readily and efficiently used in genotyping. Whenever the need to distinguish some cultivars arises, we still do not know which primer or marker to use and how many primers or reactions are needed to identify some cultivars. This is partly due to the fact that the fingerprints have not been categorized or digitized for easy reference. In fact, no efficient approach has been developed and successfully used for cultivar identification, except where phylogenetic trees and some fingerprints were employed. The former cannot tell us which information can be used or referred to for identification of a specific cultivar, while the later cannot present all the fingerprints together and is therefore not suitable for identification of many cultivars.

Developing a strategy that can make the most use of the apparent advantages of DNA markers for easy identification of pear cultivars is very vital for the pear nursery and production industry. Towards this end, we successfully identified 68 cultivars of pear using polymorphic RAPD markers by developing and deploying a new strategy. This identification could generate a CID that clearly presents the information needed for identification of groups of pear cultivars. The CID can work just like the Periodic Table of Elements is used in chemistry and is quite advantageous due to its reliability, workability, ease of use, and flexibility, since more new cultivars can be added in as they are identified. This resource would not only provide valuable information and theoretical scientific basis on cultivar identification, genetic diversity, cultivar introduction, and genetic improvement at the molecular level, but also can be an essential requirement for granting of protection to all the new varieties through DUS (distinctness, uniformity and stability) testing (Lu et al., 2009). This new approach is both efficient and practical, with less cost, rapidness and objectivity, among other attributes. This strategy can open up the immense power of DNA markers in plant cultivar identification, and the polymorphism of each primer can be used to gradually distinguish and chart every species. Although this method does not accurately reflect the genetic relationship between the cultivars/species being identified, theoretically, the earlier the separation, the greater the genetic distance between the cultivars/species, while the genetic distance between cultivars/species separated later in a CID is much closer. This method creates a readable and recordable flow chart, making fruit crop cultivar identification much easier than before.

China is an important agricultural country and has abundant plant resources, which makes the task of distinguishing plant cultivars or varieties very important. For a long time, pollen morphology, cytology and iso-enzymes have been the main techniques used for identifying pear species and cultivars (Banno et al., 2000). However, the use of DNA marker techniques is gradually gaining ground and has elicited much interest in the fruit industry (Kim et al., 2000; Teng et al., 2001; Schiliro et al., 2001; Xuan, 2008). Our new approach is not just a simple model; it also makes DNA markers more applicable for plant variety identification in practice. It has been found to be efficient in distinguishing plant cultivars and gives reliable information for future rapid identification work. Our study drastically simplifies the identifica- 
tion of plant cultivars, making it direct and as easy as measuring the length of an object using a ruler. Although a single-RAPD primer cannot distinguish all pear cultivars at the same time, this method represents a substantial increase in efficiency over previous studies. Additionally, it also reveals new evidence on the rapid identification of plant cultivars and the results further show that any two plant cultivars can be identified by the use of one specific primer. At present, we have initiated similar study to present as much identification information as possible for most fruit crop cultivars in China for the purposes of cultivar-right-protection and to provide a better service for the nursery industry.

We used RAPDs to detect many specific bands for the cultivars of the genus Pyrus, thus providing useful information for identification within this genus. It is, however, necessary to incorporate the critical limitations of this method when interpreting results. Principally, we have to consider the lack of complete reproducibility of the technique. In order to guarantee reproducibility, some adjustments in the annealing temperature, number of amplification cycles and concentrations of genomic DNA, magnesium and polymerase were made to the original program to improve the results. Therefore, it is very important to ascertain the conditions of the analysis, particularly the amount of DNA used.

This is a first report on using RAPD primers in sequence to identify pear cultivars. In order to verify the reliability of this theory, the verification of the workability and efficiency of the diagram in cultivar identification was vital and it gave satisfactory results. Therefore, this experiment suggests the possibility of utilizing DNA markers even in other plant species having a highly heterozygous genome, without the need for a genetic linkage map or any DNA sequence information to distinguish the cultivars. It appears to be an effective technique for conveniently developing selection markers in fruit cultivars, and it can also make DNA markers more applicable for plant variety identification in practice.

In conclusion, this new strategy is rapid, simple, and produces reliable results, since it was possible to demonstrate that a standard set of primers can be used to distinguish a large number of Pyrus species.

\section{ACKNOWLEDGMENTS}

Research supported by the 948 Research Fund of Ministry of Agriculture of People's Republic of China (\#2009-Z18) and the Agricultural Science and Technology Autonomous Research Fund (\#CX(10)108).

\section{REFERENCES}

Archak S, Gaikwad AB, Gautam D, Rao EVVB, et al. (2003). DNA fingerprinting of Indian cashew (Anacardium occidentale L.) varieties using RAPD and ISSR techniques. Euphytica 130: 397-404.

Baird WV, Ballard RE, Rajapakse S and Abbott AG (1996). Progress in Prunus mapping and application of molecular markers to germplasm improvement. Hortic. Sci. 31: 1099-1106.

Banno K, Liu Y, Ishikawa H, Nakano S, et al. (2000). Isozymes and RAPD markers to identify the parenthood of Japanese pear 'Kuratsuki'. J. Jpn. Soc. Hortic. Sci. 69: 208-213.

Baysal O, Siragusa M, Gümrükcü E, Zengin S, et al. (2010). Molecular characterization of Fusarium oxysporum $\mathrm{f}$. melongenae by ISSR and RAPD markers on eggplant. Biochem. Genet. 48: 524-537.

Belaj A, Satovic Z, Ismaili H, Panajoti D, et al. (2003). RAPD genetic diversity of Albanian olive germplasm and its relationships with other Mediterranean countries. Euphytica 130: 387-395.

Bell RL, Quamme HA, Layne REC and Skirvin RM (1996). Fruit Breeding. Vol. 1. In: Tree and Tropical Fruits (Janick J 
and Moore JN, eds.). Wiley, New York, 441-514.

Bhau BS, Medhi K, Das AP, Saikia SP, et al. (2009). Analysis of genetic diversity of Persea bombycina "Som" using RAPD-based molecular markers. Biochem. Genet. 47: 486-497.

Boronnikova SV, Kokaeva ZG, Gostimskii SA, Dribnokhodova OP, et al. (2007). Analysis of DNA polymorphism in a relict Uralian species, yellow foxglove (Digitalis grandiflora Mill.), using RAPD and ISSR markers. Genetika 43: 653-659.

Bousquet J, Simon L and Lalonde M (1990). DNA amplification from vegetative and sexual tissues of trees using polymerase chain reaction. Can. J. For. Res. 20: 254-257.

Cheng ZP and Huang HW (2009). SSR fingerprinting Chinese peach cultivars and landraces (Prunus persica) and analysis of their genetic relationships. Sci. Hortic. 120: 188-193.

Chiu TH, Pang JC, Chen MH and Tsen HY (2010). Improvement of strain discrimination by combination of RAPD with PFGE for the analysis of the swine isolates of Salmonella enterica serovar Choleraesuis. World J. Microb. Biot. 27: 465-469.

Corazza-Nunes MJ, Machado MA, Nunes WMC, Cristofani M, et al. (2002). Assessment of variability in grapefruits (Citrus paradise Macf.) and pommelos (C. maxima (Burm.) Merr.) using RAPD and SSR markers. Euphytica 126: 169-176.

D’Onofrio C, De Lorenzis G, Giordani T, Natali L, et al. (2009). Retrotransposon-based molecular markers in grapevine species and cultivars identification and phylogenetic analysis. Acta Hortic. 827: 45-52.

Demirsoy L, Demir T, Demirsoy H, Kacar YA, et al. (2008). Identification of some sweet cherry cultivars grown in Amasya by RAPD markers. Acta Hortic. 795: 147-152.

Ding XD, Lu LX, Chen XJ and Guan X (2000). Identifying litchi cultivars and evaluating their genetic relationships by RAPD markers. J. Trop. Subtrop. Bot. 8: 49-54.

Elidemir AY and Uzun I (2009). Assessment of genetic diversity of some important grape cultivars, rootstocks, and wild grapes in Turkey using RAPD markers. Acta Hortic. 827: 275-278.

Fang J, Twito T, Zhang Z and Chao CT (2006). Genetic relationships among fruiting-mei (Prunus mume Sieb. et Zucc.) cultivars evaluated with AFLP and SNP markers. Genome 49: 1256-1264.

Kafkas S, Ozkan H, Ak BE, Acar I, et al. (2006). Detecting DNA polymorphism and genetic diversity in a wide pistachio (Pistacia vera L.) germplasm: comparison of AFLP, ISSR and RAPD markers. J. Am. Soc. Hortic. Sci. 13: 522-529.

Kim CS, Lee GP, Han DH, Ryu KH, et al. (2000). Classification and identification of Pyrus pyrifolia using RAPD. J. Kor Soc. Hortic. Sci. 41: 119-124.

Lee GP, Lee CH and Kim CS (2004). Molecular markers derived from RAPD, SCAR, and the conserved 18S rDNA sequences for classification and identification in Pyrus pyrifolia and P. communis. Theor. Appl. Genet. 108: 14871491.

Lu X, Liu L, Gong Y, Zhao L, et al. (2009). Cultivar identification and genetic diversity analysis of broccoli and its related species with RAPD and ISSR markers. Sci. Hortic. 122: 645-648.

Melgarejo P, Martínez JJ, Hernández F, Martínez R, et al. (2009). Cultivar identification using 18S-28S rDNA intergenic spacer-RFLP in pomegranate (Punica granatum L.). Sci. Hortic. 120: 500-503.

Murray MG and Thompson WF (1980). Rapid isolation of high molecular weight plant DNA. Nucleic Acids Res. 8: 43214325.

Papp N, Szilvássy B, Abrankó L, Szabó T, et al. (2010). Main quality attributes and antioxidants in Hungarian sour cherries: identification of genotypes with enhanced functional properties. Int. J. Food Sci. Technol. 45: 395-402.

Qu X, Lu J and Lamikanra O (1996). Genetic diversity in Muscadine and American bunch grapes based on randomly amplified polymorphic DNA (RAPD) analyses. J. Am. Soc. Hortic. Sci. 121: 1020-1023.

Saker MM, Adawy SS, Mohamed AA and El-Itriby HA (2006). Monitoring of cultivar identity in tissue culture-derived date palms using RAPD and AFLP analysis. Biol. Plant. 50: 198-204.

Schiliro E, Predieri S and Bertaccini A (2001). Use of random amplified polymorphic DNA analysis to detect genetic variation in Pyrus species. Plant. Mol. Biol. Rep. 19: 271-272.

Silvestrini M, Maluf MP, Silvarolla MB, Guerreiro-Filho O, et al. (2008). Genetic diversity of a Coffea germplasm collection assessed by RAPD markers. Genet. Res. Crop. Evol. 55: 901-910.

Stark-Urnau M (2002a). Use of RAPD-markers in Malus x domestica (apple) and Pyrus communis (pear) for cultivar identification - Part I: Malus x domestica (apple). Erwerbsobstbau 44: 139-144.

Stark-Urnau M (2002b). Use of RAPD-markers in Malus x domestica (apple) and Pyrus communis (pear) for cultivar identification - Part II: Pyrus communis (Birne). Erwerbsobstbau 44: 167-171.

Teng Y, Tanabe K, Tamura F and Itai A (2001). Genetic relationships of pear cultivars in Xinjiang, China, as measured by RAPD markers. J. Hortic. Sci. Biotechnol. 76: 771-779.

Williams JG, Kubelik AR, Livak KJ, Rafalski JA, et al. (1990). DNA polymorphisms amplified by arbitrary primers are useful as genetic markers. Nucleic Acids Res. 18: 6531-6535. 
Xuan H (2008). Identifying European pear (Pyrus communis L.) cultivars at the KOB by using apple SSRs. Acta Hortic. 800: 439-445.

Yonemoto Y, Chowdhury AK, Kato H and Macha MM (2006). Cultivars identification and their genetic relationships in Dimocarpus longan subspecies based on RAPD markers. Sci. Hortic. 109: 147-152. 